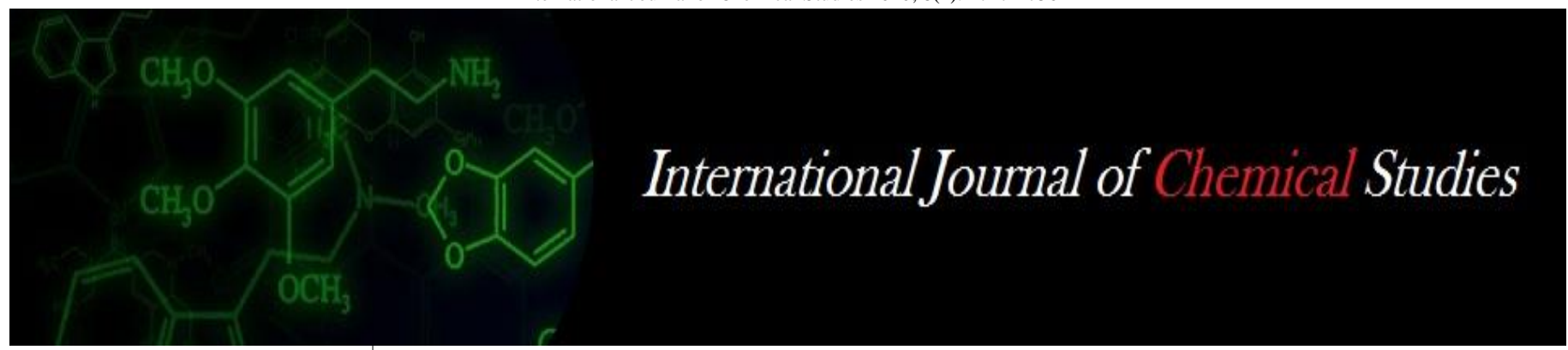

P-ISSN: 2349-8528

E-ISSN: 2321-4902

www.chemijournal.com

IJCS 2020; 8(2): 2747-2756

(C) 2020 IJCS

Received: 22-01-2020

Accepted: 24-02-2020

\section{SH Peerzada}

Division of Plant Pathology,

Sher-e-Kashmir University of

Agricultural Sciences and

Technology-Srinagar

(SKUAST-K), Jammu and

Kashmir India

HS Viswanath

Division of Plant Pathology, Sher-e-Kashmir University of Agricultural Sciences and

Technology-Srinagar

(SKUAST-K), Jammu and

Kashmir India

\section{KA Bhat}

Division of Plant Pathology,

Sher-e-Kashmir University of

Agricultural Sciences and

Technology-Srinagar

(SKUAST-K), Jammu and

Kashmir India
Corresponding Author: SH Peerzada

Division of Plant Pathology, Sher-e-Kashmir University of Agricultural Sciences and Technology-Srinagar (SKUAST-K), Jammu and Kashmir India

\section{Impact of seed dressing and systemic fungicidal sprays in controlling the magnitude and spread of the late blight (Phytophthora infestans) disease in addition to augmenting the yield of superior grade potato tubers}

\author{
SH Peerzada, HS Viswanath and KA Bhat
}

DOI: https://doi.org/10.22271/chemi.2020.v8.i2ap.9167

\begin{abstract}
Potato late blight is one of the devastating plant diseases. The pathogen is primarily seed borne and polycyclic in nature dispersing many secondary sporangiospores from foliar infected plants to healthy ones spreading rapidly within short span causing heavy yield losses. So, in the present study, two different experiments were conducted separately, where in the first one, seed dressing with different fungicides was carried out under pot conditions followed by second experiment in finding the effect of systemic foliar fungicidal sprays under field conditions against the incidence and intensity of late blight disease. Four different fungicides (one protectant and three systemic) viz., mancozeb 75 WP, dimethomorph 50WP, cymoxanil $8+$ mancozeb $64 \mathrm{WP}$ and metalaxyl $8+$ mancozeb $64 \mathrm{WP}$, were evaluated as seed dressers against late blight (Phytophthora infestans) of potato cv. Kufri Jyoti in pot experiments during 2016 and 2017cropping seasons. The results obtained during both cropping seasons revealed that all the fungicidal seed dressers delayed the appearance of first symptoms of the disease. The disease incidence and intensity recorded indicated that all the fungicides significantly reduced the late blight at all the periods compared to control (water). The 'A'(AUDPC) values were significantly reduced when tubers were treated with different fungicides. Tuber treatment with dimethomorph or metalaxyl + mancozeb showed minimum 'A' value of 125.91 compared to the maximum "A" value of 185.20 for undressed control during 2016 and similar trend of results was observed during 2017 cropping season. In the second experiment of systemic foliar fungicidal sprays, three systemic fungicides viz. dimethomorph $50 \mathrm{WP}$ at $0.05 \%(\mathrm{C} 1), 0.10 \%(\mathrm{C} 2)$ and $0.15 \%(\mathrm{C} 3)$ concentrations, metalaxyl 8+mancozeb $64 \mathrm{WP}$ and Cymoxanil $8+$ mancozeb 72 WP at $0.15 \%(\mathrm{C} 1), 0.20 \%(\mathrm{C} 2) \& 0.25 \%(\mathrm{C} 3)$ concentrations sprayed at ten days interval after symptom development till dehaulming stage were evaluated against the disease incidence and intensity during 2016 \& 2017 cropping seasons. The results obtained during 2016 cropping season revealed that, among the systemic/translaminar fungicidal foliar sprays, all the fungicides were equally superior to water-sprayed control in reducing the blight incidence from 74.04 to17.03 per cent. On an average, foliar spray with dimethomorph yielded the minimum blight intensity of 11.64 per cent followed by cymoxanil 8 + mancozeb 72 WP 13.02 per cent spray compared to 56.27 per cent obtained in water- sprayed check. Dimethomorph foliar sprays at C2 and C3 concentrations were found most effective recording the lowest blight intensities of 8.38 and $8.44 \%$ respectively and least "A" value followed by foliar spray with cymoxanil 8+mancozeb 72 WP (10.35-10.41\%) compared to water sprayed check (56.27\%) during 2016 and similar trend was observed during 2017 cropping season. It was also found that the foliar sprays with dimethomorph resulted in the highest tuber yield $(206.14 \mathrm{q}$ tubers per hectare) and large sized tubers followed by foliar sprays with metalaxyl 8+mancozeb 64 WP as compared to water-sprayed control plots (152.72 q/ ha)with small sized tubers.
\end{abstract}

Keywords: Late blight of potato, seed dressing, foliar sprays, systemic fungicides, disease intensity, tuber yield

\section{Introduction}

Potato (Solanum tuberosum L.) is one of the most important food crops worldwide which represents a valuable source of nutrients in a balanced diet. In terms of human consumption, the potato is the third most important food crop in the world, following only rice and wheat. Potato is also one of the important food crops in India. 
India stands second in world's potato production where it is cultivated over an area of 2.13 million hectares with a production of 43.77 million metric tonnes. The area and production of the Potato crop in Jammu \& Kashmir state are 6.9 thousand hectares and 1.27 lakh metric tonnes, respectively (Viswanath et al., 2020) ${ }^{[15]}$. However, its yield and quality is adversely affected by frequent occurrence of a number of fungal, bacterial and viral diseases, among which late blight [Phytophthora infestans (Mont) de-Bary] is highly destructive. Reports of complete field destruction due to late blight epidemics are relatively common. The fungus is responsible for global annual crop loss of US \$ 12 billion. Yield loss due to late blight in India varies from year to year and range from $20-75 \%$. The pathogen produces water soaked lesions with chlorotic borders that are small at first but expand rapidly under humid conditions, blighting the entire plant in only a few days with subsequent rotting of the developing tubers resulting in heavy yield losses under favourable conditions each year with reduction in global production by approximately 15 per cent. Losses of up to 10 to 75 per cent by the disease have been reported in India (Peerzada et al., 2020b) ${ }^{[10]}$.

Phytophthora infestans, an oomycetes fungi is primarily seed and soil borne in nature. Although, controlling late blight has become increasingly demanding because of the evolution of new races by gradually developing resistance against available chemical fungicides, among all disease controlling strategies, chemical control proved to be the most effective one in reducing the intensity of disease and crop yield losses. Fungicides used for disease control were generally classified based on their nature in two types i.e systemic/translaminar fungicides and protectant (contact) fungicides. Protectant fungicides are prophylactic in nature, which are applied before the occurrence of the disease further checking the symptom development. Most of protectant fungicides eliminate the fungi that come in contact with them. Whereas, systemic fungicides are taken up by a plant and then translocated within plant system and therefore, can be applied even after disease appearance at any stage of disease development. As pathogen is primarily seed borne in nature, it is necessary to protect the disease infection at this stage itself. So, in the present study, seed dressing with different fungicides was primarily taken up to find its impact on the overall disease reduction. Secondly, during the growth phase of the crop, disease spread is very rapid due to the polycyclic nature of the pathogen, so use of prophylactic contact fungicidal sprays can curb the disease only to a certain extent and cannot stop the spread. So, apart from the seed dressing in this study, very importantly systemic fungicidal sprays at ten days intervals after symptom development until dehaulming stage of potato crop was also taken up to assess the amount of disease reduction as well as the increase in tuber grade, quality and yield of potato.

\section{Material and methods}

Isolation of pathogen and production of inoculum for conducting seed dressing experiment in pots

Blighted samples were brought to the laboratory and the isolation of pure culture was made on V-8 agar medium using standard plant pathological technique. A pure culture of $P$. infestans was transferred onto V8 medium amended with rose Bengal in $90 \mathrm{~mm}$ diameter Petri dishes and incubated for about 14 days. The obtained culture is mass multiplied on sand-maize medium (Muthusamy, 1972) ${ }^{[7]}$.

\section{Evaluation of different fungicides as seed dressers under pot conditions}

The efficacy of fungitoxicants viz; mancozeb 75WP, dimethomorph $50 \mathrm{WP}$, cymoxanil $8+$ Mancozeb $64 \mathrm{WP}$, metalaxyl $8+$ Mancozeb $64 \mathrm{WP}$, as seed dressing was evaluated in earthen pots $(30 \mathrm{~cm}$ diameter) arranged in completely randomized design at experimental farm of Division of Plant Pathology, SKUAST-Kashmir, with five pots containing two plants each representing a treatment and each treatment replicated thrice. Sandy loam soil collected from the field was sterilized at $1.05 \mathrm{~kg} \mathrm{~cm}^{-2}$ pressure for two hours and filled in pots. The late blight pathogen culture on sand maize medium was added to soil @ 1:20 (w/w) of the pathogens and soil, three days before sowing. Tubers dressed separately with different fungicides were sown in pathogen infested soil and the undressed tubers planted in pots served as control. Healthy whole seed tubers of uniform size of cultivar kufri Jyoti, were put in pots at the depth of 1 to $2 \mathrm{~cm}$, $15 \mathrm{~cm}$ apart and at least $2.5 \mathrm{~cm}$ from the edge of the pots. The per cent disease incidence and intensity were recorded periodically at 10 days interval, after the first appearance of disease in control pots, and the disease progress curve was calculated.

\section{Evaluation of systemic fungicides as foliar sprays under field conditions}

The experiment was carried out in the field at SKUASTKashmir, wadura during 2016 and 2017 cropping seasons in randomized block design with three replicates, planting the tubers in last week of March 2016 and 1ST week of April 2017 by adopting the recommended package of practices for raising the crop (Anonymous, 2004) ${ }^{[1]}$. Irrigations were given at interval of three weeks after planting the tubers. The field was sub-divided into plots of size $2 \mathrm{~m} \mathrm{x} 2 \mathrm{~m}$ with 50 plants at a spacing $20 \times 40 \mathrm{~cm}$ with one meter border between the plots and the blocks to minimize fungicide drift and limit inter-plot interference. Three systemic/translaminar fungicides viz. cymoxanil $8+$ mancozeb $72 \mathrm{WP}$, metalaxyl $8+$ mancozeb 64 WP at $0.15 \%(\mathrm{C} 1), 0.20 \%(\mathrm{C} 2) \& 0.25 \%(\mathrm{C} 3)$ concentrations, whereas Dimethomorph $50 \mathrm{WP}$ at $0.05 \%$ (C1), $0.10 \%(\mathrm{C} 2)$ and $0.15 \%$ concentrations were evaluated for their effectiveness against late blight under field conditions The spray application was initiated at the first appearance of the disease symptoms and repeated till dehaulming with 10 days intervals ensuring complete coverage of upper and lower leaf and shoot- surface. Late blight was assessed at 10 days intervals from the $1^{\text {st }}$ fungicidal spray and continued till dehaulming adopting the procedure given under Data recorded on late blight intensity was used to compute area under disease progress curve "AUDPC "as per the scale mentioned for the disease intensity. Tubers were dug out at maturity when all the vines were dead; the yield data was recorded from individual plots and converted into qtls/ha and the tubers categorized into different grades depending on tuber diameter

\begin{tabular}{|c|c|}
\hline Tuber diameter $(\mathbf{c m})$ & Tuber grade \\
\hline $8 \mathrm{~cm}$ and above & $\mathrm{A}$ \\
\hline $4 \mathrm{~cm}$ to $8 \mathrm{~cm}$ & $\mathrm{~B}$ \\
\hline $4 \mathrm{~cm}$ and below & $\mathrm{C}$ \\
\hline
\end{tabular}

The per cent yield increase (PYI) was calculated as:

Tuber yield obtained with - Tuber yield obtained in Fungicide treatment control plots Tuber yield obtained in control 
Disease incidence: An area of $1 \mathrm{~m}$ x $1.5 \mathrm{~m}$ was randomly marked at 10 different places in the field for recording the total number of diseased and healthy plants at flowering stage and 10 days before dehaulming of the crop. Mathematically,

\section{Late blight incidence $(\%)=\frac{\mathrm{n}}{\mathrm{N}} \times 100$}

Where $\mathrm{n}$ is the number of plants showing blight symptoms and $\mathrm{N}$ the total number of plants examined. An average of the ten assessments in the fields represented the average disease incidence of the field.

Disease intensity: An area of $1 \mathrm{~m} \times 1.5 \mathrm{~m}$ was randomly marked at 10 different places in the field and the observation on the extent of the foliage blighted was recorded at flowering stage and 10 days before dehaulming using the disease rating scale given by Mohan and Thind (1999) ${ }^{[6]}$

\begin{tabular}{|c|c|}
\hline Disease Score & $\begin{array}{c}\text { Score description in terms of foliage infected } \\
(\boldsymbol{\%})\end{array}$ \\
\hline 0 & No visible symptoms \\
\hline 1 & $1-10$ \\
\hline 2 & $11-25$ \\
\hline 3 & $26-50$ \\
\hline 4 & $51-75$ \\
\hline 5 & $>75$ \\
\hline
\end{tabular}

The disease intensity was calculated by using the following formula

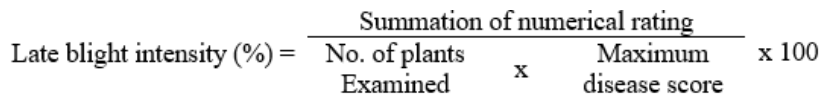

Per cent disease intensity recorded was transformed using arc sine transformation and subjected to analysis of variance (ANOVA) as suggested by Gomez and Gomez (1984) ${ }^{[3]}$

The Area under disease progress curve (AUDPC) "A" value: The intensity of foliar blight was determined at 10 days interval from the onset of first symptoms until the end of vegetation period (dehaulming) and expressed in per cent of the infected leaf area as per the scale mentioned above in the disease intensity. The Area under disease progress curve (AUDPC) " $A$ " value was determined as per the method given by Shanner and Finney (1997) using the formula. The AUDPC value was calculated with the following formula

$$
=\sum_{\mathrm{i}=1}^{\mathrm{n}-1} \underset{2}{\left.\left(\mathrm{X}_{i}+\mathrm{X}_{i}+1\right)\right]} \underset{2}{\left(\mathrm{~T}_{\mathrm{i}}+1-\mathrm{T}_{\mathrm{i}}\right)}
$$

Where $\mathrm{X}=\%$ Disease intensity at different dates $(\mathrm{X} 1+\mathrm{X} 2$, $\mathrm{X} 2+\mathrm{X} 3, \mathrm{X} 3+\mathrm{X} 4 \ldots$ and so on)

$\mathrm{T}=$ Time interval between two observations

$\mathrm{n}=$ Total number of observations

\section{Results and Discussion \\ Evaluation as seed dressers}

Four different fungicides viz., mancozeb 75 WP, dimethomorph 50WP, cymoxanil $8+$ mancozeb $64 \mathrm{WP}$ and metalaxyl $8+$ mancozeb $64 \mathrm{WP}$, were evaluated as seed dressers against late blight (Phytophthora infestans) of potato cv. Kufri Jyoti in pot experiments during 2016 and 2017 cropping seasons. The results obtained during 2016 cropping season (Table-1) revealed that all the fungicidal seed dressers delayed the appearance of first symptoms of the disease. The first initial symptoms appeared 37 days after planting with tubers treated dimethomorph $50 \mathrm{WP}$ followed by those of metalaxyl $8+$ mancozeb 64 WP and cymoxanil 8 + mancozeb 64 WP 35 days compared to untreated check in which case first symptoms appeared only 29 days after planting. The disease incidence and intensity recorded indicated that all the fungicides significantly reduced the late blight at all the periods compared to check, such that a terminal disease incidence and intensity of 83.33 and 35.00 , in control, were reduced to 50.00 and 21.55 per cent, respectively, in tubers treated with Metalaxyl $8+$ Mancozeb 64 WP. The 'A' values were significantly reduced when tubers were treated with different fungicides. Tuber treatment with dimethomorph or metalaxyl + mancozeb showed minimum " $A$ ' value of 125.91 compared to the maximum " $A$ " value of 185.20 for undressed control. Similarly, same trend was observed during 2017 cropping season. The results obtained during the cropping season (Table-2) revealed that all the fungicidal seed dressers delayed the appearance of initial symptom of the disease. The first initial symptoms appeared 34 days after planting of dimethomorph treated tubers followed by metalaxyl + mancozeb and cymoxanil +mancozeb treated tubers (33 days) compared to untreated check in which case the first symptoms appeared only 27 days after planting. The disease incidence and intensity recorded indicated that all the fungicides significantly reduced the late blight incidence at all the periods compared to check such that a terminal disease incidence and intensity of 86.66 and 35.90 per cent, were reduced to 56.66 and 22.15 per cent, respectively, by tuber treatments with metalyxyl 8 and mancozeb 64 WP. The area under disease progress curve was minimum (128.13) in case of tuber dressed with either

\begin{tabular}{|c|c|c|c|c|c|c|c|c|}
\hline \multirow{2}{*}{ Treatment } & \multirow{2}{*}{$\begin{array}{c}\text { Days taken to } 1^{\text {st }} \\
\text { symptom appearance }\end{array}$} & \multicolumn{3}{|c|}{ Incidence (\%) days after planting } & \multicolumn{4}{|c|}{ Intensity (\%) days after planting } \\
\hline & & 50 & 60 & 70 & 50 & 60 & 70 & "A" Value \\
\hline Mancozeb 75 WP & 33 & $\begin{array}{l}10.00 \\
(18.43)\end{array}$ & $\begin{array}{c}26.66 \\
(30.78)\end{array}$ & $\begin{array}{c}53.33 \\
(47.00) \\
\end{array}$ & $\begin{array}{c}6.80 \\
(8.95)\end{array}$ & $\begin{array}{l}14.90 \\
(22.26)\end{array}$ & \begin{tabular}{|l}
26.45 \\
$(30.52)$
\end{tabular} & 157.62 \\
\hline Dimethomorph $50 \mathrm{WP}$ & 37 & $\begin{array}{c}10.00 \\
(18.43)\end{array}$ & $\begin{array}{c}30.00 \\
(33.00)\end{array}$ & $\begin{array}{c}50.00 \\
(45.00)\end{array}$ & $\begin{array}{c}5.80 \\
(11.29)\end{array}$ & $\begin{array}{c}12.10 \\
(20.17)\end{array}$ & $\begin{array}{c}22.90 \\
(28.52)\end{array}$ & 132.25 \\
\hline Cymoxanil $8+$ Mancozeb 64 WP & 35 & $\begin{array}{c}6.66 \\
(12.29)\end{array}$ & $\begin{array}{c}33.33 \\
(35.00)\end{array}$ & $\begin{array}{c}53.33 \\
(46.92)\end{array}$ & $\begin{array}{c}6.95 \\
(9.05)\end{array}$ & $\begin{array}{l}14.10 \\
(21.99)\end{array}$ & $\begin{array}{l}24.70 \\
(29.59)\end{array}$ & 149.62 \\
\hline Metalaxyl $8+$ Mancozeb 64 WP & 35 & $\begin{array}{c}3.33 \\
(6.14)\end{array}$ & $\begin{array}{c}33.33 \\
(34.22)\end{array}$ & $\begin{array}{c}50.00 \\
(45.22)\end{array}$ & $\begin{array}{c}5.30 \\
(15.67)\end{array}$ & $\begin{array}{c}10.70 \\
19.08)\end{array}$ & $\begin{array}{l}21.55 \\
(27.53)\end{array}$ & 125.91 \\
\hline Control & 29 & $\begin{array}{c}23.33 \\
(28.78)\end{array}$ & $\begin{array}{c}63.33 \\
(52.86)\end{array}$ & $\begin{array}{c}83.33 \\
(66.14)\end{array}$ & $\begin{array}{l}10.10 \\
(17.71)\end{array}$ & $\begin{array}{l}20.60 \\
(26.62)\end{array}$ & $\begin{array}{c}35.00 \\
(35.91)\end{array}$ & 185.20 \\
\hline \multicolumn{2}{|l|}{ Mean } & 10.66 & 37.33 & $58.00(50.01)$ & 7.41 & 14.48 & 26.12 & - \\
\hline
\end{tabular}
metalaxyl +mancozeb compared to ' $A$ ' value of 228.08 obtained in undressed tubers.

Table 1: Evaluation of fungicides against late blight (Phytophthora infestans) as seed dresser of potato cv. Kufri Jyoti planted in pots during 2016 cropping season 


\begin{tabular}{|c|c|c|c|c|c|c|c|}
\hline & $(16.81)$ & $(37.17)$ & & $(12.53)$ & $(22.07)$ & $(30.41)$ & \\
\hline S.Em \pm & 0.5 & 0.54 & 0.91 & 0.7 & 0.64 & 0.83 & 00.76 \\
\hline $\mathrm{CD}(0.05)$ & NS & 1.84 & 2.43 & NS & 1.72 & 2.11 & 10.41 \\
\hline
\end{tabular}

Tubers planted on $20^{\text {th }}$ March (2016);

Data are mean of 5 replications

Table 2: Evaluation of fungicides against late blight (Phytophthora infestans) as seed dresser of potato cv. Kufri Jyoti planted in pots during 2017 cropping season

\begin{tabular}{|c|c|c|c|c|c|c|c|c|}
\hline \multirow[b]{2}{*}{ Treatment } & \multirow{2}{*}{$\begin{array}{c}\text { Days taken to } 1^{\text {st }} \text { symptom } \\
\text { appearance }\end{array}$} & \multicolumn{3}{|c|}{ Incidence $(\%)$ days after planting } & \multicolumn{4}{|c|}{ Intensity (\%) days after planting } \\
\hline & & 50 & 60 & 70 & 50 & 60 & 70 & $\begin{array}{l}\text { "A" } \\
\text { Value }\end{array}$ \\
\hline Mancozeb 75 WP & 32 & $\begin{array}{l}13.33 \\
(21.14)\end{array}$ & $\begin{array}{l}30.00 \\
(33.00)\end{array}$ & $\begin{array}{c}60.00 \\
(58.85)\end{array}$ & $\begin{array}{c}7.40 \\
(15.54)\end{array}$ & $\begin{array}{l}14.65 \\
(22.23)\end{array}$ & $\begin{array}{c}27.10 \\
(31.30)\end{array}$ & 159.49 \\
\hline Dimethomorph $50 \mathrm{WP}$ & 34 & $\begin{array}{c}13.33 \\
(17.71)\end{array}$ & $\begin{array}{c}36.66 \\
(36.84)\end{array}$ & $\begin{array}{c}56.66 \\
(48.93)\end{array}$ & $\begin{array}{c}6.30 \\
(14.38)\end{array}$ & $\begin{array}{l}12.10 \\
(20.23)\end{array}$ & $\begin{array}{l}23.30 \\
(28.67)\end{array}$ & 134.51 \\
\hline $\begin{array}{c}\text { Cymoxanil } 8+\text { Mancozeb } \\
64 \mathrm{WP}\end{array}$ & 34 & $\begin{array}{c}10.00 \\
(15.00)\end{array}$ & $\begin{array}{c}43.33 \\
(41.07)\end{array}$ & $\begin{array}{c}60.00 \\
(53.06)\end{array}$ & $\begin{array}{c}7.45 \\
(15.53)\end{array}$ & $\begin{array}{c}14.10 \\
(21.94)\end{array}$ & $\begin{array}{c}25.30 \\
(29.98)\end{array}$ & 159.24 \\
\hline $\begin{array}{c}\text { Metalaxyl 8+ Mancozeb } 64 \\
\text { WP }\end{array}$ & 33 & $\begin{array}{c}13.33 \\
(21.14) \\
\end{array}$ & $\begin{array}{c}33.33 \\
(34.92) \\
\end{array}$ & $\begin{array}{c}56.66 \\
(51.14) \\
\end{array}$ & $\begin{array}{c}5.80 \\
(13.51)\end{array}$ & $\begin{array}{c}10.70 \\
(19.03) \\
\end{array}$ & $\begin{array}{c}22.15 \\
(27.98) \\
\end{array}$ & 128.13 \\
\hline Control & 27 & $\begin{array}{l}26.66 \\
(30.99) \\
\end{array}$ & $\begin{array}{l}66.66 \\
(54.99) \\
\end{array}$ & $\begin{array}{c}86.66 \\
(72.29) \\
\end{array}$ & $\begin{array}{l}12.10 \\
(19.89) \\
\end{array}$ & $\begin{array}{l}21.60 \\
(27.53) \\
\end{array}$ & $\begin{array}{c}35.90 \\
(36.72) \\
\end{array}$ & 228.08 \\
\hline \multicolumn{2}{|c|}{ Mean } & $\begin{array}{l}15.33 \\
(21.19)\end{array}$ & $\begin{array}{c}42.00 \\
(40.16)\end{array}$ & $\begin{array}{c}65.33 \\
(55.25)\end{array}$ & $\begin{array}{c}7.81 \\
(15.77)\end{array}$ & $\begin{array}{c}14.63 \\
(22.19)\end{array}$ & $\begin{array}{c}26.75 \\
(30.93)\end{array}$ & - \\
\hline \multicolumn{2}{|c|}{ S.Em \pm} & 0.5 & 0.54 & 0.91 & 0.7 & 0.64 & 0.83 & \\
\hline \multicolumn{2}{|c|}{$\mathrm{CD}(0.05)$} & 2.41 & 1.84 & 2.43 & 2.01 & 1.72 & 2.11 & \\
\hline
\end{tabular}

Tubers planted on March $4^{\text {th }} 2017$

Data are mean of 5 replications

\section{Evaluation of Systemic/translaminar fungicides as foliar sprays}

\section{Effect on late blight development}

The different fungicidal foliar sprays made separately at periodical intervals on potato crop cv. Kufri Jyoti were evaluated during 2016 and 2017 cropping seasons for their efficacy in controlling potato late blight with concomitant effects on tuber yield. The results obtained during 2016 cropping season revealed that, among systemic/translaminar fungicidal foliar sprays (Table-3), all the fungicides viz dimethomorph $50 \mathrm{WP}$, metalaxyl 8+mancozeb $64 \mathrm{WP}$ and Cymoxanil $8+$ mancozeb 72 WP were equally superior to water - sprayed check in reducing the blight incidence from 74.04 to17.03 per cent, the $\mathrm{C} 2$ and $\mathrm{C} 3$ concentrations proving equally effective compared to $\mathrm{C} 1$ concentration, in general. There exists a significant interaction between fungicides and their concentrations. All the three test fungicides at C2 and C3 concentration reduced the disease incidence to 13.33-33.33 per cent from 75.55 per cent observed in water sprayed check, respectively.
On an average, foliar spray with dimethomorph yielded the minimum blight intensity of 11.64 per cent followed by cymoxanil $8+$ mancozeb 72 WP 13.02 per cent spray compared to 56.27 per cent obtained in water- sprayed check. Among concentrations, in general, the higher concentration of C2 and C3 exhibited lower disease intensity of 22.02-22.07 per cent than the lower $\mathrm{C} 1$ concentration where 28.24 per cent blight intensity was observed. A significant interaction between fungicides and their concentrations in containing the blight intensity also recorded. Dimethomorph foliar spray at $\mathrm{C} 2$ and $\mathrm{C} 3$ concentration was the most effective fungicide recording the lowest blight intensity of 8.38 and 8.44 per cent followed by foliar spray with cymoxanil $8+$ mancozeb $72 \mathrm{WP}$ (10.35-10.41\%) compared to water sprayed check $(56.27 \%)$.

The area under disease progress curve ("A" value), computed on the basis of the periodical assessment of blight significantly varied between treatments. On an overall mean basis, dimethomorph 50 WP foliar sprays showed the least "A" value of 46.38 per cent

Table 3: Effect of foliar sprays with systemic/translaminar fungicides on progress of late blight (Phytophthora infestans) of potato cv.Kufri Jyoti recorded at 10 days interval during 2016 cropping season

\begin{tabular}{|c|c|c|c|c|c|c|c|c|c|c|c|c|}
\hline \multirow[t]{2}{*}{ Fungicide } & \multicolumn{4}{|c|}{$\begin{array}{c}\text { Late blight incidence }(\%) \text { at fungicide } \\
\text { concentration }\end{array}$} & \multicolumn{4}{|c|}{$\begin{array}{c}\text { Late blight intensity (\%) at fungicide } \\
\text { concentration }\end{array}$} & \multicolumn{4}{|c|}{ 'A' value } \\
\hline & C1 & C2 & C3 & Mean & C1 & C2 & C3 & Mean & C1 & C2 & $\mathrm{C3}$ & Mean \\
\hline $\begin{array}{c}\text { Cymoxanil } 8+ \\
\text { Mancozeb } 72 \mathrm{WP}\end{array}$ & $\begin{array}{c}33.33 \\
(35.19)\end{array}$ & $\begin{array}{l}19.99 \\
(26.35)\end{array}$ & $\begin{array}{c}19.99 \\
(26.35)\end{array}$ & $\begin{array}{c}24.41 \\
(29.30)\end{array}$ & $\begin{array}{c}18.31 \\
(25.26)\end{array}$ & $\begin{array}{c}10.41 \\
(18.71)\end{array}$ & $\begin{array}{c}10.35 \\
(18.72)\end{array}$ & $\begin{array}{c}13.02 \\
(20.90)\end{array}$ & 72.01 & 42.03 & 39.10 & 51.05 \\
\hline $\begin{array}{c}\text { Metalaxyl8+ Mancozeb } \\
64 \text { WP } \\
\end{array}$ & $\begin{array}{c}33.33 \\
(35.19) \\
\end{array}$ & $\begin{array}{c}19.99 \\
(26.35) \\
\end{array}$ & $\begin{array}{r}19.99 \\
(26.35) \\
\end{array}$ & $\begin{array}{r}24.33 \\
(29.22) \\
\end{array}$ & $\begin{array}{l}20.30 \\
(26.74) \\
\end{array}$ & $\begin{array}{c}13.16 \\
(21.08) \\
\end{array}$ & $\begin{array}{l}13.10 \\
(21.18)\end{array}$ & $\begin{array}{c}15.52 \\
(23.00)\end{array}$ & 100.52 & 69.52 & 70.38 & 80.14 \\
\hline Dimethomorph $50 \mathrm{WP}$ & $\begin{array}{c}24.44 \\
(29.58) \\
\end{array}$ & $\begin{array}{l}13.33 \\
(20.97)\end{array}$ & $\begin{array}{l}13.33 \\
(20.97)\end{array}$ & $\begin{array}{c}17.03 \\
(23.84) \\
\end{array}$ & $\begin{array}{l}18.11 \\
(25.17)\end{array}$ & $\begin{array}{c}8.44 \\
(16.76)\end{array}$ & $\begin{array}{c}8.38 \\
(16.77)\end{array}$ & $\begin{array}{c}11.64 \\
(19.57)\end{array}$ & 65.56 & 37.21 & 36.37 & 46.38 \\
\hline Control(water spray) & $\begin{array}{c}73.33 \\
(59.02)\end{array}$ & $\begin{array}{c}73.33 \\
(59.33)\end{array}$ & $\begin{array}{c}75.55 \\
(60.92)\end{array}$ & $\begin{array}{c}74.07 \\
(59.65)\end{array}$ & $\begin{array}{c}56.27 \\
(48.72)\end{array}$ & $\begin{array}{c}56.27 \\
(48.72) \\
\end{array}$ & $\begin{array}{c}56.27 \\
(48.72)\end{array}$ & $\begin{array}{c}56.27 \\
(48.72)\end{array}$ & 282.73 & 283.0 & 284.67 & 283.47 \\
\hline Mean & $\begin{array}{c}41.10 \\
(39.74) \\
\end{array}$ & $\begin{array}{c}31.66 \\
(33.17) \\
\end{array}$ & $\begin{array}{c}32.13 \\
(33.59) \\
\end{array}$ & & $\begin{array}{c}28.24 \\
(31.47) \\
\end{array}$ & $\begin{array}{c}22.07 \\
(26.32) \\
\end{array}$ & $\begin{array}{c}22.02 \\
(26.35) \\
\end{array}$ & & 130.21 & 107.94 & 107.63 & \\
\hline & \multicolumn{2}{|c|}{ S.Em \pm} & \multicolumn{2}{|c|}{$\mathrm{CD}(\mathrm{P}=0.05 \%)$} & \multicolumn{2}{|c|}{ S.Em \pm} & \multicolumn{2}{|c|}{$\mathrm{CD}(\mathrm{P}=0.05 \%)$} & \multicolumn{2}{|c|}{ S.Em \pm} & \multicolumn{2}{|c|}{$\mathrm{CD}(\mathrm{P}=0.05 \%)$} \\
\hline Fungicide $(\mathrm{F})$ & \multicolumn{2}{|c|}{0.60} & \multicolumn{2}{|c|}{1.62} & \multicolumn{2}{|c|}{0.55} & \multicolumn{2}{|c|}{1.10} & \multicolumn{2}{|c|}{1.40} & \multicolumn{2}{|c|}{9.86} \\
\hline Concentration (C) & \multicolumn{2}{|l|}{0.78} & \multicolumn{2}{|c|}{2.53} & \multirow{2}{*}{\multicolumn{2}{|c|}{0.85}} & \multicolumn{2}{|c|}{1.74} & \multicolumn{2}{|c|}{1.90} & \multicolumn{2}{|c|}{11.94} \\
\hline $\mathrm{F} \times \mathrm{C}$ & \multicolumn{2}{|c|}{1.40} & \multicolumn{2}{|c|}{4.37} & & & \multicolumn{2}{|c|}{2.71} & \multicolumn{2}{|c|}{2.48} & \multicolumn{2}{|c|}{18.61} \\
\hline
\end{tabular}


Figures in parenthesis are arc sine transformed values. Spray started with the first appearance of disease on May $8^{\text {th }} 2016$ and repeated at after every 10 days interval. Cymoxanil $8+$ Mancozeb 72 WP and Metalaxyl $8+$ Mancozeb 64 WP evaluated at $0.15 \%(\mathrm{C} 1), 0.20 \%(\mathrm{C} 2) \& 0.25 \%(\mathrm{C} 3)$, whereas Dimethomorph $50 \mathrm{WP}$ evaluated at $0.05 \%$ (C1), $0.10 \%(\mathrm{C} 2)$ and $0.15 \%$ concentrations followed by cymoxanil $8+$ mancozeb 72 WP yielding " $\mathrm{A}$ " value of 51.05 compared to 283.47 " $A$ " value of obtained in water sprayed check plots. In general, the higher test concentrations of C2-C3 yielded the minimum (107.63-107.94) for the disease as compared to their lower (C1) concentration (130.21). A significant interaction also operated between different fungicides and their concentrations with regard to their effects on AUDPC. Foliar spray with dimethomorph at C2-C3 concentration exhibited the lowest "A "value of 36.37-37.21 followed by foliar sprays with both cymoxanil $8+$ mancozeb 72 WP and or (39.10-42.03) as compared to 282.73-284.67 obtained in water sprayed check.

Similar trend was observed during 2017 field experiments. The results reveal that, among systemic/translaminar fungicidal foliar sprays (Table-4), indicate that all the fungicides viz., dimethomorph 50 WP, metalaxyl 8+mancozeb $64 \mathrm{WP}$ and cymoxanil 8+mancozeb $72 \mathrm{WP}$, were equally superior to water- sprayed check in reducing the blight incidence from 73.33 to 18.51 per cent. There exists a significant interaction between fungicides and their concentrations. All the three test fungicides at $\mathrm{C} 2-\mathrm{C} 3$ concentration reduced the disease incidence to 15.55 per cent from 73.33 per cent observed in water-sprayed check.

On an average, foliar spray with dimethomorph $50 \mathrm{WP}$ yielded the minimum blight intensity of 9.59 per cent followed by cymoxanil 8+mancozeb 72 WP (12.09\%) compared to 56.28 per cent obtained in water- sprayed check. Among concentrations, in general, the higher concentration of C2 and C3 exhibited lower disease intensity of 21.08 and 21.42 per cent than the lower concentration $(\mathrm{C} 1)$ where 27.14 per cent. A significant interaction between fungicides and their concentrations in containing the blight intensity also existed. Dimethomorph $50 \mathrm{WP}$ foliar spray at C2 and C3 concentration was the most effective fungicide recording the lowest blight intensity of 6.01-6.05 per cent followed by foliar spray with cymoxanil $8+$ mancozeb $72 \mathrm{WP}$ at the same.

Table 4: Effect of foliar sprays with systemic/translaminar fungicides on progress of late blight (Phytophthora infestans) of potato cv.Kufri Jyoti recorded at 10 days interval during 2017 cropping season

\begin{tabular}{|c|c|c|c|c|c|c|c|c|c|c|c|c|}
\hline \multirow[t]{2}{*}{ Fungicide } & \multicolumn{4}{|c|}{$\begin{array}{l}\text { Late blight incidence }(\%) \text { at fungicide } \\
\text { concentration }\end{array}$} & \multicolumn{4}{|c|}{$\begin{array}{l}\text { Late blight intensity }(\%) \text { at fungicide } \\
\text { concentration }\end{array}$} & \multicolumn{4}{|c|}{ “A" value } \\
\hline & C1 & C2 & C3 & Mean & C1 & $\mathrm{C2}$ & C3 & Mean & C1 & $\mathrm{C2}$ & $\mathbf{C 3}$ & Mean \\
\hline $\begin{array}{c}\text { Cymoxanil 8+ Mancozeb } \\
72 \%\end{array}$ & $\begin{array}{c}31.11 \\
(33.87)\end{array}$ & $\begin{array}{l}19.99 \\
(26.35)\end{array}$ & $\begin{array}{c}19.99 \\
(26.35)\end{array}$ & $\begin{array}{l}23.70 \\
(28.86)\end{array}$ & $\begin{array}{l}16.80 \\
(24.16)\end{array}$ & $\begin{array}{c}9.77 \\
(17.91)\end{array}$ & $\begin{array}{c}9.71 \\
(18.72)\end{array}$ & $\begin{array}{l}12.09 \\
(20.15)\end{array}$ & 76.16 & 43.54 & 42.58 & 54.09 \\
\hline $\begin{array}{c}\text { Metalaxyl8+ Mancozeb 64 } \\
\text { WP }\end{array}$ & $\begin{array}{c}31.11 \\
(33.87)\end{array}$ & $\begin{array}{c}19.99 \\
(26.35)\end{array}$ & $\begin{array}{c}19.99 \\
(26.35)\end{array}$ & $\begin{array}{l}23.70 \\
(28.86\end{array}$ & $\begin{array}{c}18.72 \\
(25.53)\end{array}$ & $\begin{array}{c}12.83 \\
(20.95)\end{array}$ & $\begin{array}{c}13.01 \\
(21.91)\end{array}$ & $\begin{array}{l}14.90 \\
(22.57\end{array}$ & 95.60 & 68.11 & 67.79 & 77.17 \\
\hline Dimethomorph $50 \mathrm{WP}$ & $\begin{array}{l}20.00 \\
(26.65)\end{array}$ & $\begin{array}{l}15.55 \\
(23.31)\end{array}$ & $\begin{array}{l}15.55 \\
(23.31)\end{array}$ & $\begin{array}{l}18.51 \\
(25.35)\end{array}$ & $\begin{array}{l}16.71 \\
(24.12)\end{array}$ & $\begin{array}{c}6.05 \\
(14.21)\end{array}$ & $\begin{array}{c}6.01 \\
(14.21)\end{array}$ & $9.59(17.50$ & 60.05 & 26.66 & 26.32 & 37.68 \\
\hline Control (water spray) & $\begin{array}{c}73.33 \\
(59.02)\end{array}$ & $\begin{array}{c}73.33 \\
(59.02)\end{array}$ & $\begin{array}{c}73.33 \\
(59.02)\end{array}$ & $\begin{array}{c}73.33 \\
(59.02)\end{array}$ & $\begin{array}{c}56.34 \\
(48.78)\end{array}$ & $\begin{array}{c}55.61 \\
(48.33)\end{array}$ & $\begin{array}{c}56.89 \\
(49.15)\end{array}$ & $\begin{array}{c}56.28 \\
(48.75)\end{array}$ & 292.10 & 293.90 & 291.45 & 292.48 \\
\hline \multirow[t]{2}{*}{ Mean } & $\begin{array}{c}38.88 \\
(38.33\end{array}$ & $\begin{array}{c}32.21 \\
(33.17)\end{array}$ & $\begin{array}{c}33.33 \\
(34.52)\end{array}$ & & $\begin{array}{c}27.14 \\
(30.65)\end{array}$ & $\begin{array}{c}21.08 \\
(25.42)\end{array}$ & $\begin{array}{c}21.42 \\
(25.66)\end{array}$ & & 130.97 & 101.05 & 107.03 & \\
\hline & \multicolumn{2}{|c|}{ S.Em \pm} & \multicolumn{2}{|c|}{$\mathrm{CD}(\mathrm{P}=0.05 \%)$} & \multicolumn{2}{|c|}{ S.Em \pm} & \multicolumn{2}{|c|}{$\mathrm{CD}(\mathrm{P}=0.05 \%)$} & \multicolumn{2}{|c|}{ S.Em \pm} & \multicolumn{2}{|c|}{$\mathrm{CD}(\mathrm{P}=0.05 \%)$} \\
\hline Fungicide (F) & \multicolumn{2}{|c|}{0.96} & \multicolumn{2}{|c|}{5.37} & \multicolumn{2}{|c|}{0.85} & \multicolumn{2}{|c|}{1.34} & \multicolumn{2}{|c|}{6.12} & \multicolumn{2}{|c|}{24.09} \\
\hline Concentration (C) & \multicolumn{2}{|c|}{1.12} & \multicolumn{2}{|c|}{3.93} & \multicolumn{2}{|c|}{0.98} & \multicolumn{2}{|c|}{2.30} & \multirow{2}{*}{\multicolumn{2}{|c|}{4.21}} & \multicolumn{2}{|c|}{19.04} \\
\hline $\mathrm{F} \times \mathrm{C}$ & \multicolumn{2}{|c|}{2.11} & \multicolumn{2}{|c|}{8.17} & \multicolumn{2}{|c|}{1.27} & \multicolumn{2}{|c|}{6.89} & & & \multicolumn{2}{|c|}{24.63} \\
\hline
\end{tabular}

Figures in parenthesis are arc sin transformed values. Spray started with the first appearance of disease on May $12^{\text {th }} 2017$ and repeated at after every 10 days interval. Disease incidence and intensity recorded at 10 days intervals. Cymoxanil 8+mancozeb $72 \mathrm{WP}$ and metalaxyl 8+ mancozeb $64 \mathrm{WP}$ evaluated at $0.15 \%(\mathrm{C} 1), 0.20 \%$ (C2) \& $0.25 \%$ (C3), whereas Dimethomorph $50 \mathrm{WP}$ evaluated at $0.05 \%(\mathrm{C} 1), 0.10 \%(\mathrm{C} 2)$ and $0.15 \%(\mathrm{C} 3)$ concentrations

concentration of C2-C3 yielding blight intensity of 9.71-9.77 per cent compared to water sprayed check (53.30\%).

The area under disease progress curve ("A" value), computed on the basis of periodical assessment of blight significantly varied between treatments. On an overall mean basis, dimethomorph 50 WP foliar sprays showed the least "A" value of 37.86 followed by cymoxanil $8+$ mancozeb 72 WP and metalaxyl $8+$ mancozeb 64 WP foliar sprays yielding "A" value of 54.09 and 77.17 , respectively compared to 292.48 obtained in water- sprayed check plots. In general, the higher test concentration of $\mathrm{C} 2$ and $\mathrm{C} 3$ yielded the minimum " $\mathrm{A}$ " Valve (107.03- 108.05) for the disease as compared to their lower (C1) concentration of (130.97). A significant interaction also operated between different fungicides and their concentration with regard to their effects on AUDPC. Foliar spray with dimethomorph $50 \mathrm{WP}$ at C3 and C2 concentrations exhibited the lowest "A "value of 26.32 and 26.66 followed by foliar sprays with both cymoxanil $8+$ mancozeb $72 \mathrm{WP}$ and metalaxyl 8+mancozeb $64 \mathrm{WP}$ at the same concentration yielding "A "value of 42.58-43.54 and 67.79-77.17 compared to $291.45-293.90$ obtained in water sprayed check.

\section{Effect on tuber yield}

The foliar sprays with different systemic/translaminar fungicides during 2016 also resulted in significant differences in tuber yield (Table-5). On an overall mean basis, the foliar sprays with dimethomorph resulted in the highest tuber yield of $229.19 \mathrm{q} / \mathrm{ha}$ followed by foliar sprays with metalaxyl 8+mancozeb 64 WP which yielded 206.14 q tubers per hectare as compared to only $152.72 \mathrm{q} /$ ha obtained in watersprayed control plots. On an average, the higher concentration (C2-C3) proved superior in providing tuber yields of 210.28$210.60 \mathrm{q} / \mathrm{ha}$ compared to $171.58 \mathrm{q}$ obtained at $\mathrm{C} 1$ concentration. A significant interaction between fungicides and their concentrations existed in promoting tuber yields. Foliar sprays with dimethomorph at $\mathrm{C} 2-\mathrm{C} 3$ concentrations resulted in the maximum tuber yield of 245.68-260.54 q/ha followed by cymoxanil 8+mancozeb. 
Table 5: Effect of foliar sprays with systemic/translaminar fungicides on tuber yield (Phytophthora infestans) of potato cv. Kufri Jyoti during 2016 cropping season

\begin{tabular}{|c|c|c|c|c|c|c|c|c|}
\hline \multirow{2}{*}{ Fungicide } & \multicolumn{8}{|c|}{ Tuber yield $\left(\mathrm{q} \mathrm{ha}^{-1}\right)$ at fungicide concentrationPer cent yield gain over control at fungicide concentration } \\
\hline & \begin{tabular}{|l|l|}
$\mathrm{C1}$ \\
\end{tabular} & $\mathrm{C2}$ & C3 & Mean & C1 & $\mathrm{C2}$ & C3 & Mean \\
\hline Cymoxanil 8 + Mancozeb 72 WP & 170.21 & 210.52 & 225.00 & 201.91 & 11.84 & 36.35 & 48.41 & 32.20 \\
\hline Metalaxyl8+ Mancozeb 64 WP & 182.60 & 215.68 & 220.13 & 206.14 & 19.98 & 39.69 & 45.20 & 34.95 \\
\hline Dimethomorph 50 WP & 181.34 & 260.54 & 245.68 & 229.19 & 19.16 & 68.75 & 62.05 & 49.98 \\
\hline Control (water spray) & 152.18 & 154.39 & 151.60 & 152.72 & -- & -- & -- & \\
\hline \multirow[t]{2}{*}{ Mean } & 171.58 & 210.28 & 210.60 & & & & & \\
\hline & \multicolumn{2}{|c|}{ S.Em \pm} & \multicolumn{2}{|c|}{$\mathrm{CD}(\mathrm{p}=0.05)$} & & & & \\
\hline Fungicide (F) & \multicolumn{2}{|c|}{1.66} & \multicolumn{2}{|c|}{7.01} & & & & \\
\hline Concentration (C) & \multicolumn{2}{|c|}{1.99} & \multicolumn{2}{|c|}{11.06} & & & & \\
\hline $\mathrm{F} \times \mathrm{C}$ & \multicolumn{2}{|c|}{2.01} & \multicolumn{2}{|c|}{16.20} & & & & \\
\hline
\end{tabular}

Spray started with the first appearance of disease on May $8^{\text {th }}$ 2016 and repeated at after every 10 days interval. Disease incidence/intensity recorded at 10 days interval. Cymoxanil 8 + Mancozeb 72 WP and Metalaxyl $8+$ Mancozeb 64 WP evaluated at $0.15 \%(\mathrm{C} 1), 0.20 \%(\mathrm{C} 2) \& 0.25 \%(\mathrm{C} 3)$, whereas Dimethomorph $50 \mathrm{WP}$ evaluated at $0.05 \%(\mathrm{C} 1), 0.10 \%(\mathrm{C} 2)$ and $0.15 \%(\mathrm{C} 3)$ concentrations

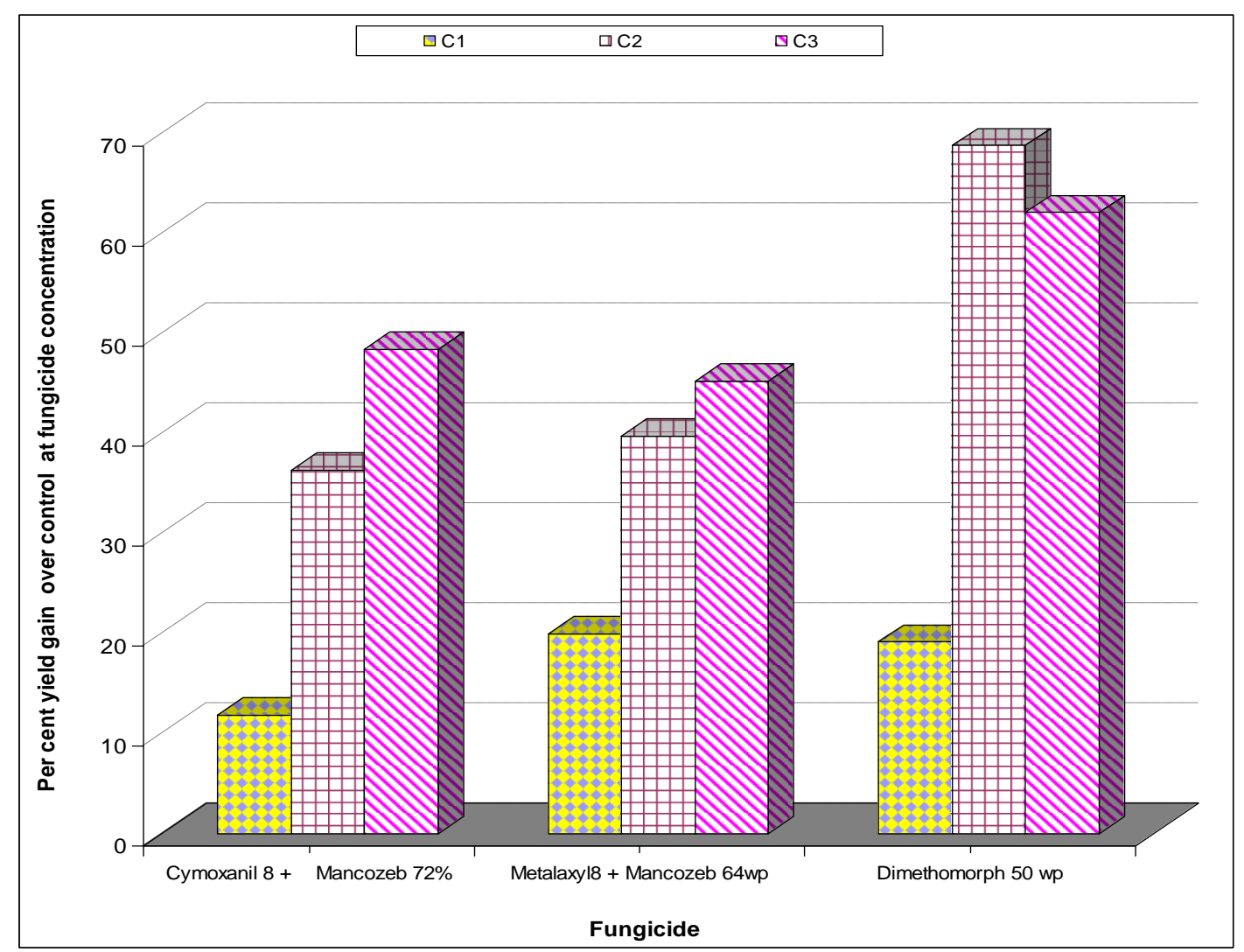

Fig 1: Effect of foliar sprays with systemic/translaminar fungicides on tuber yield (Phytophthora infestans) of potato cv. Kufri Jyoti during 2016 cropping season

$72 \mathrm{WP}$ at the same concentrations was the next best treatment showing tuber yield of 210.52-225.00 q/ha. An insight into the data (Fig. 1) reveals 62 to 69 per cent gain in tuber yields with the foliar spray of either dimethomorph or chlorothalonil at $\mathrm{C} 2-\mathrm{C} 3$ concentration over water sprayed check followed by metalaxyl 8+mancozeb 64 WP foliar sprays which yielded a gain of only 40-45 per cent.

The fungicidal sprays were further found to significantly influence the size/grade of tubers (Table-6). On an average, foliar sprays with the test fungicides (dimethomorph, metalaxyl $8+$ mancozeb 64 WP or cymoxamil $8+$ mancozeb 72 WP), significantly increased the proportion of A and B grade tubers to 35.07-37.45 and 50.16-55.67 per cent, respectively from 20.86 and 37.94 per cent obtained in water- sprayed check. The proportion of unmarketable $\mathrm{C}$ grade tubers was, on an average, significantly less (14.92-16.42\%) in treatments sprayed with any of the fungicides compared to check $(27.97 \%)$. A significant interaction between fungicides and their concentrations in improving the tuber grade also existed. Foliar sprays with metalaxyl 8+mancozeb $64 \mathrm{WP}$ and cymoxanil $8+$ mancozeb $72 \mathrm{WP}$ at $\mathrm{C} 2$ concentration yielded maximum of 47.17-49.87 per cent. A grade tubers, followed by foliar sprays with metalaxyl $8+$ mancozeb $64 \mathrm{WP}$ at $\mathrm{C} 3$ concentration which yielded 40.31 per cent A grade tubers compared to 21.46-22.15 per cent A grade tubers obtained in water sprayed check plots. Similarly, the foliar sprays with cymoxanil 8+mancozeb 72 WP metaxyle $8+$ mancozeb 64 WP at $\mathrm{C} 2$ concentration yielded 61.17 to 58.75 per cent $\mathrm{B}$ grade tubers compared to check (37.14-52.14\%). An insight into the Fig. 2 indicates that the proportion of marketable (A and $B$ grade) tuber yield was significantly increased by spraying any of the fungicides. The unmarketable yield was 
noticeably decreased by spraying these fungicides. The results obtained on the yield of potato tubers during 2017 are presented in (Table-7). On an overall mean basis, foliar spray with cymoxamil $8+$ mancozeb $72 \mathrm{WP}$ resulted in the highest tuber yield of $216.58 \mathrm{q} / \mathrm{ha}$ followed by foliar sprays with dimethomorph and metalaxyl 8+mancozeb 64 WP which yielded 214.15-199.78

Table 6: Effect of foliar sprays with Systemic/translaminar fungicides on tuber grade of potato cv. Kufri Jyoti during 2016 cropping season

\begin{tabular}{|c|c|c|c|c|c|c|c|c|c|c|c|c|}
\hline \multirow{3}{*}{ Fungicide } & \multicolumn{9}{|c|}{ Tuber grade (\%) at fungicide concentration } & \multirow{2}{*}{\multicolumn{3}{|c|}{ Mean (\%) }} \\
\hline & \multicolumn{3}{|c|}{ C1 } & \multicolumn{3}{|c|}{$\mathrm{C2}$} & \multicolumn{3}{|c|}{ C3 } & & & \\
\hline & $\mathbf{A}$ & B & C & $\mathbf{A}$ & B & $\mathbf{C}$ & $\mathbf{A}$ & B & C & $\mathbf{A}$ & B & C \\
\hline Cymoxanil 8 + Mancozeb 72 WP & 22.46 & 50.76 & 26.76 & 49.87 & 61.17 & 12.63 & 40.02 & 55.09 & 6.21 & 37.45 & 55.67 & 5.20 \\
\hline Metalaxyl8+ Mancozeb 64 WP & 23.43 & 50.32 & 26.23 & 47.17 & 58.75 & 12.20 & 40.31 & 48.82 & 10.85 & 36.97 & 52.63 & 16.42 \\
\hline Dimethomorph 50 WP & 25.03 & 47.25 & 27.71 & 40.03 & 52.48 & 7.98 & 40.15 & 50.75 & 9.09 & 35.07 & 50.16 & 14.92 \\
\hline Control (water spray) & 18.99 & 52.81 & 28.19 & 21.46 & 52.14 & 26.39 & 22.15 & 53.89 & 23.82 & 20.86 & 37.94 & 27.97 \\
\hline \multirow[t]{2}{*}{ Mean } & 22.47 & 50.26 & 27.11 & 39.63 & 56.13 & 14.80 & 35.65 & 52.16 & 12.46 & & & \\
\hline & & \multicolumn{2}{|c|}{ S.Em+ } & \multicolumn{2}{|c|}{$\mathrm{CD}(\mathrm{p}=0.05)$} & & & & & & & \\
\hline Fungicide $(\mathrm{F})$ & & \multicolumn{2}{|c|}{0.41} & \multicolumn{2}{|c|}{$(1.45)$} & & & & & & & \\
\hline Concentration $(\mathrm{C})$ & & \multicolumn{2}{|c|}{0.69} & \multicolumn{2}{|c|}{$(2.51)$} & & & & & & & \\
\hline $\mathrm{F} \times \mathrm{C}$ & & \multicolumn{2}{|c|}{1.14} & \multicolumn{2}{|c|}{$(2.90)$} & & & & & & & \\
\hline
\end{tabular}

Spray started with the first appearance of disease on May $12^{\text {th }}$ 2016 and repeated at after every 10 days interval. Disease incidence/intensity recorded at 10 days interval. Cymoxanil $8+$ mancozeb 72WP and metalaxyl $8+$ mancozeb 64WP evaluated at $0.15 \%(\mathrm{C} 1), 0.20 \%(\mathrm{C} 2) \& 0.25 \%$ (C3), whereas Dimethomorph 50 WP evaluated at $0.05 \%(\mathrm{C} 1), 0.10 \%(\mathrm{C} 2)$ and $0.15 \%$ concentration.

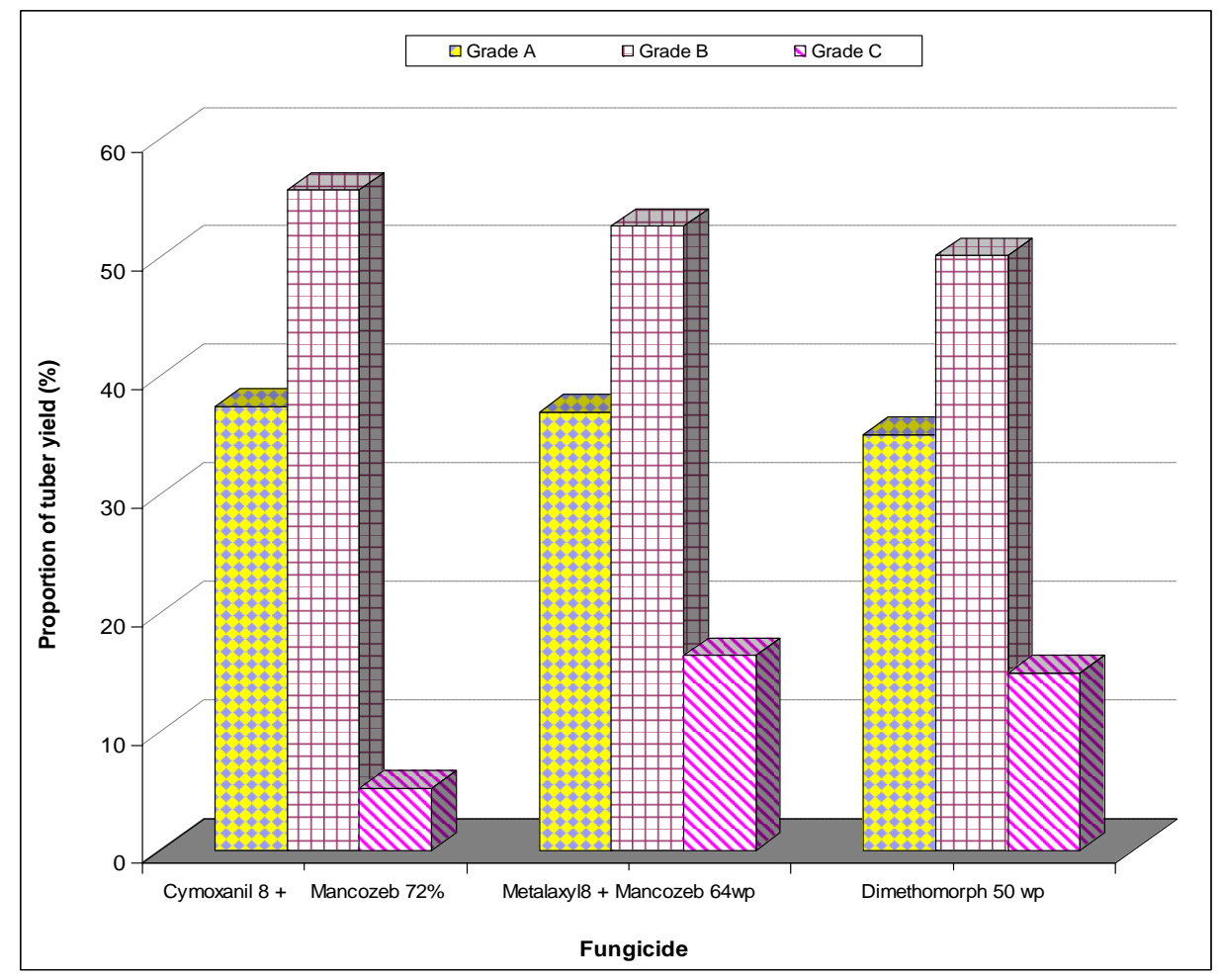

A and $\mathrm{B}$ grade marketable yield, $\mathrm{C}$ is unmarketable yield

Fig 2: Proportion of marketable and unmarketable grade obtained by spraying different fungicides during 2016 cropping season

Table 7: Effect of foliar sprays with systemic/translaminar fungicides on tuber yield (Phytophthora infestans) of potato cv. Kufri Jyoti during 2017 cropping season

\begin{tabular}{|c|c|c|c|c|c|c|c|c|}
\hline \multirow{2}{*}{ Fungicide } & \multicolumn{7}{|c|}{ Tuber yield $\left(\mathbf{q ~ h a}^{-1}\right)$ at fungicide concentration Per cent yield gain over control at fungicide concentration } \\
\cline { 2 - 9 } & $\mathbf{C 1}$ & $\mathbf{C 2}$ & $\mathbf{C 3}$ & Mean & C1 & C2 & C3 & Mean \\
\hline Cymoxanil 8 + Mancozeb 72 WP & 166.30 & 223.33 & 260.10 & 216.58 & 13.39 & 52.96 & 78.15 & 48.16 \\
\hline Metalaxyl8+ Mancozeb 64 WP & 178.04 & 212.15 & 209.16 & 199.78 & 21.40 & 45.30 & 43.26 & 36.65 \\
\hline Dimethomorph 50 WP & 175.83 & 229.55 & 237.08 & 214.15 & 19.89 & 57.22 & 62.38 & 46.49 \\
\hline Control (water spray) & 146.65 & 146.00 & 146.00 & 146.22 & - & - & - & \\
\hline Mean & 166.71 & 202.76 & 213.09 & & & & & \\
\hline & Sem \pm & $\mathrm{CD}(\mathrm{P}=0.05)$ & & Sem \pm & $\mathrm{CD}(\mathrm{P}=0.05)$ & $\mathrm{Sem} \pm$ & $\mathrm{CD}(\mathrm{P}=0.05)$ \\
\hline Fungicide (F) & 0.81 & 4.18 & & 2.01 & 7.21 & 1.34 & 7.81 \\
\hline Concentration (C) & 1.21 & 7.72 & & 2.35 & 10.56 & 1.92 & 11.41 \\
\hline F x C & 3.12 & 12.29 & & 2.94 & 15.44 & 3.28 & 13.77 \\
\hline
\end{tabular}


Spray started with the first appearance of disease on May $12^{\text {th }}$ 2017 and repeated at after every 10 days interval. Disease incidence/intensity recorded at 10 days interval. Fungicide sprayed at 10 intervals. . Cymoxanil 8+mancozeb 72WP and metalaxyl $8+$ mancozeb 64WP evaluated at $0.15 \%$ (C1), $0.20 \%$ (C2) \& $0.25 \%$ (C3), whereas Dimethomorph 50 WP evaluated at $0.05 \% \quad(\mathrm{C} 1), \quad 0.10 \% \quad(\mathrm{C} 2)$ and $0.15 \%$ concentration

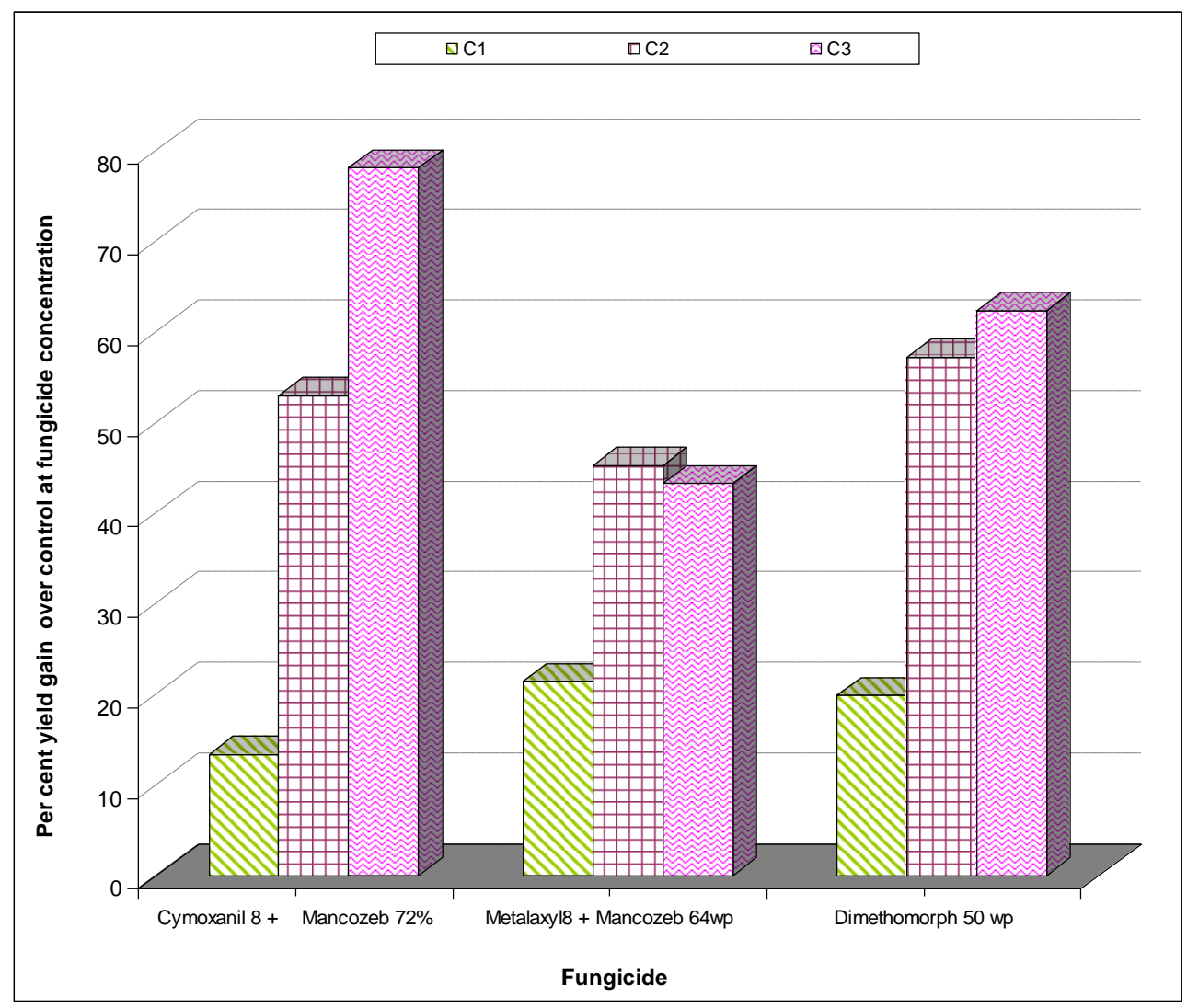

Fig 3: Effect of foliar sprays with systemic/translaminar fungicides on tuber yield (Phytophthora infestans) of potato cv. Kufri Jyoti during 2017 cropping season

q tubers per hectare as compared to only $146.22 \mathrm{q} /$ ha obtained in water- sprayed control plots. On an average, the higher concentrations (C2-C3) proved superior providing tuber yields of 202.76-213.09 q/ha compared to $166.71 \mathrm{q} / \mathrm{ha}$ obtained at $\mathrm{C} 1$ concentration. A significant interaction between fungicides and their concentrations existed in promoting tuber yields. Foliar sprays with dimethomorph 50WP at C2-C3 concentration resulted in the maximum tuber yield of 229.55-237.08 q/ha, followed by cymoxanil $8+$ mancozeb 72 WP (223.33-2630.10 q/ha). Foliar spray with metalaxyl $8+$ mancozeb $64 \mathrm{WP}$ at the same concentrations yielded 209.16-212.15 q/ha. An insight into the data (Fig. 3) reveals 62.40 to 57.22 per cent gain in tuber yields by the foliar spray with dimethomorph at $\mathrm{C} 2-\mathrm{C} 3$ concentration over water- sprayed check followed by cymoxanil 8+mancozeb 72 WP foliar sprays at the same concentration which yielded a gain of only 52.96-78.15 per cent.

The fungicidal sprays were further found to significantly influence the tuber size/grade (Table-8). On an average, foliar sprays with all the test fungicides viz., dimethomorph, metalaxyl $8+$ mancozeb 64 WP or cymoxanil $8+$ mancozeb 72 WP, significantly increased the proportion of A and B grade tubers to $35.48-38.84$ and $46.50-49.57$ per cent, respectively from 16.26 and 37.02 per cent, respectively, obtained in water- sprayed check. The proportion of unmarketable C grade tubers was, on an average, significantly less (18.27$18.40 \%$ ) in treatments sprayed with any of the fungicides compared to check $(45.78 \%)$. A significant interaction between fungicides and their concentrations in improving the tuber grade also existed. Foliar sprays with metalaxyl $8+$ mancozeb $64 \mathrm{WP}$ and cymoxanil $8+$ mancozeb $72 \mathrm{WP}$ (C2 concentration) yielded maximum of 47.66 per cent A grade tubers followed by foliar sprays with cymoxanil $8+$ mancozeb $72 \mathrm{WP}$ at C2 concentration which yielded maximum of 43.95 per cent A grade tubers compared to 17.39 per cent A grade tubers obtained in water-spray check. Similarly, foliar sprays with dimethomorph 75WP and cymoxanil 8+mancozeb 72 $\mathrm{WP}$ at $\mathrm{C} 2$ and $\mathrm{C} 3$ concentration provided maximum proportion (48.63 and 58.91) B grade tubers compared to check (35.84-36.00\%).

Table 8: Effect of foliar sprays with systemic/translaminar fungicides on tuber grade of potato cv. Kufri Jyoti during 2017 cropping season

\begin{tabular}{|c|c|c|c|c|c|c|c|c|c|c|c|c|}
\hline \multirow{3}{*}{ Fungicide } & \multicolumn{9}{|c|}{ Tuber grade (\%) at fungicide concentration } & \multirow{2}{*}{\multicolumn{3}{|c|}{ Mean $(\%)$}} \\
\hline & \multicolumn{3}{|c|}{ C1 } & \multicolumn{3}{|c|}{$\mathbf{C 2}$} & \multicolumn{3}{|c|}{$\mathbf{C 3}$} & & & \\
\hline & $\mathbf{A}$ & B & $\mathbf{C}$ & $\mathbf{A}$ & B & C & $\mathbf{A}$ & B & $\mathbf{C}$ & $\mathbf{A}$ & B & $\mathbf{C}$ \\
\hline Cymoxanil 8+ Mancozeb 72 WP & 27.90 & 46.12 & 25.97 & 43.95 & 48.63 & 12.67 & 40.30 & 49.45 & 10.24 & 37.38 & 46.33 & 16.29 \\
\hline Metalaxyl8+ Mancozeb 64WP & 28.75 & 40.13 & 31.22 & 47.66 & 58.91 & 12.58 & 40.11 & 49.46 & 10.42 & 38.84 & 49.57 & 18.40 \\
\hline Dimethomorph 50WP & 25.67 & 40.14 & 34.18 & 39.90 & 48.85 & 11.23 & 40.87 & 50.52 & 9.41 & 35.48 & 46.50 & 18.27 \\
\hline Control (water spray) & 13.43 & 39.24 & 47.32 & 17.39 & 36.00 & 45.95 & 17.97 & 35.84 & 44.12 & 16.26 & 37.02 & 45.78 \\
\hline Mean & 23.93 & 45.15 & 30.92 & 37.22 & 51.84 & 16.85 & 34.81 & 50.42 & 14.94 & & & \\
\hline
\end{tabular}




\begin{tabular}{|c|c|c|c|c|c|c|c|c|c|c|}
\hline & & S.Em+ & $\mathrm{CD}(\mathrm{p}=0.05)$ & & & & & & & \\
\hline Fungicide (F) & & 0.31 & $(1.06)$ & & & & & & & \\
\hline Concentration (C) & & 0.42 & $(1.83)$ & & & & & & & \\
\hline F x C & & 0.68 & $(2.12)$ & & & & & & & \\
\hline
\end{tabular}

Spray started with the first appearance of disease on May $12^{\text {th }}$ 2017 and repeated at after every 10 days interval. Disease incidence/intensity recorded at 10 days interval. Cymoxanil 8+mancozeb 72WP and metalaxyl 8+mancozeb 64WP evaluated at $0.15 \%(\mathrm{C} 1), 0.20 \%(\mathrm{C} 2) \& 0.25 \%(\mathrm{C} 3)$, whereas Dimethomorph 50 WP evaluated at $0.05 \%(\mathrm{C} 1), 0.10 \%$ (C2) and $0.15 \%$ concentration.

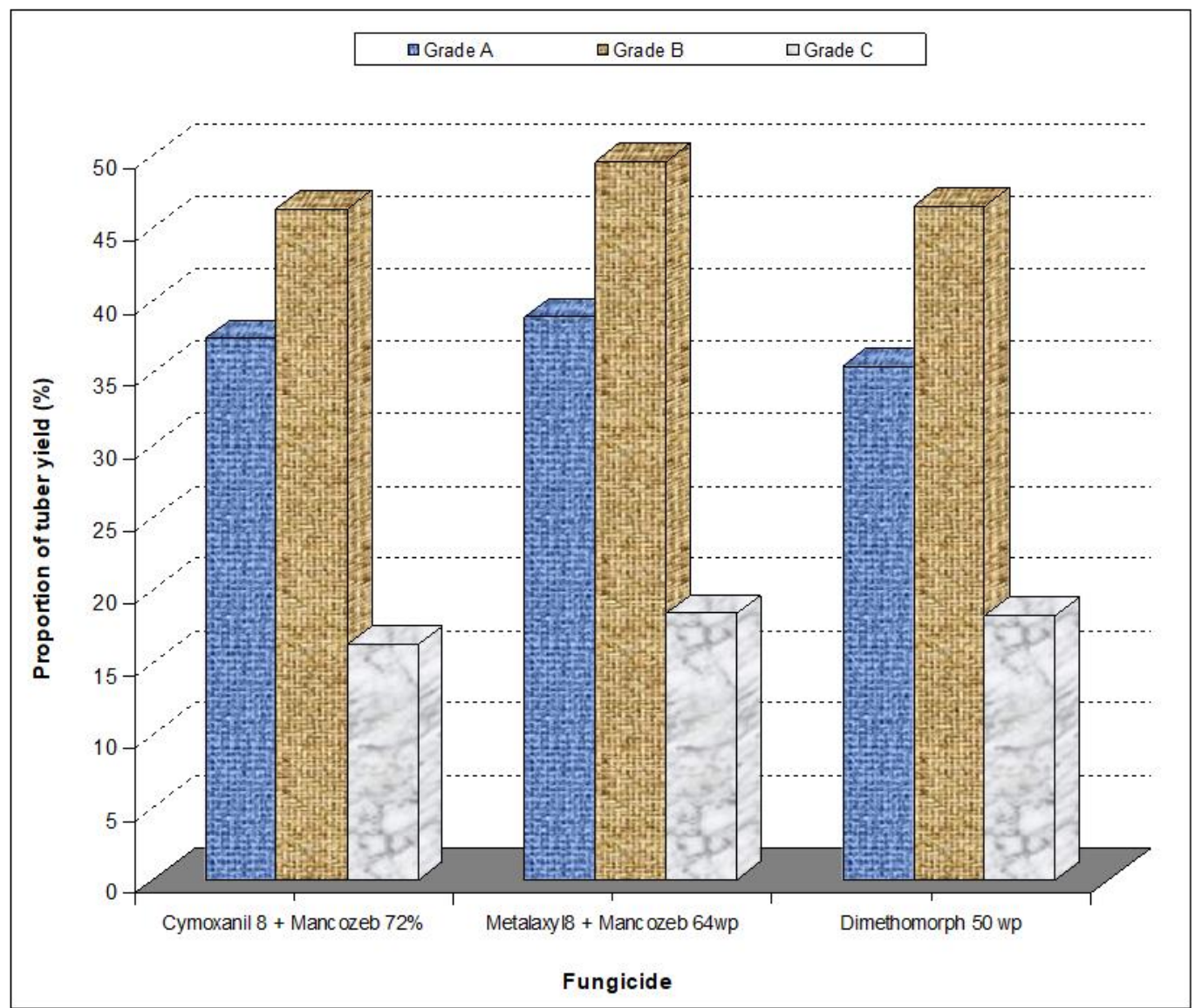

A and B grade marketable yield, $\mathrm{C}$ is unmarketable yield

Fig 4: Proportion of marketable and unmarketable grade obtained by spraying different fungicides during 2017 cropping season

An insight into the data Fig. 4 indicate that the proportion of marketable tuber yield was significantly increased by spraying any of fungicides. The unmarketable yield was noticeably decreased by spraying these fungicides

The use of fungicides for controlling plant diseases caused by fungi are well documented (Nene and Thapliyal, 1993); however, the control of diseases such as that of potato late blight caused by Phycomycetous fungi, by the use of traditional protectant fungicide has not been so successful necessitating to evaluate newer molecules for its control. The in vivo evaluation was, therefore, made by using systemic chemicals as tuber dressers or as foliar sprays at recommended concentrations. Tuber dressing with dimethomorph or metalaxyl $8+$ mancozeb 64WP delayed the initial symptoms of the disease to 37 and 34 days compared to check in which case initial symptom of the disease appeared only 29 and 27 days after planting in both the years 2016 and 2017, respectively, the treatments resulting in corresponding decrease in terminal disease intensity and A values. Since the pathogen is known to perpetuate in potato tubers, the tuber treatments with effective fungicides especially the translaminar/systemic ones is likely to inhibit or eradicate mycelial growth in tubers resulting in delayed initial appearance. The reduction in initial inoculum and the systemicity of the effective translaminar fungicides could explain the reduced terminal disease intensity (Porter, 2006 ; Kankwatsa et al., 2002) ${ }^{[4]}$. The late blight manifests mainly as foliar blight which spreads and kills the foliage by producing sporangia and zoospores. The application of fungicides that would help to inhibit mycelial growth and act as antisporulants are likely to control the spread of this disease In an attempt to evaluate the test systemic/translaminar fungicides as foliar sprays, dimethomorph followed by cymoxanil $8+$ mancozeb $72 \mathrm{WP}$ provided minimum disease and maximum gain in yield. The present studies are in accordance with those of many researchers who found that dimethomorph, cymoxanil and metaxyl have shown better mycelial inhibition against the pathogen (Peerzada et al., 2020a) " ${ }^{\text {[9] }}$. Chakraborty and Mazumdar, $2012^{[2]}$ reported that the formulation containing mancozeb and any one of the effective systemic/translaminar fungicides such as Cymoxanil or metalaxyl seem to provide better field control of late blight. They also reported that the severe late blight can be effectively managed with prophylactic spray of mancozeb @0.25\%followed by cymoxanil + mancozeb or dimethomorph +mancozeb @ $0.3 \%$ 
at the onset of disease. Khadka et al., $2016^{[5]}$ also reported that spraying with Dimethomorph and fenamidon+mancozeb showed less disease against late blight of potato. Siddique et al., 2016 reported that the highest percentage of disease control and the highest yield were recorded with Cymoxanil $8 \%+$ Mancozeb $64 \%$ fungicide which are in consonance with our results. The yield gains with the improvement in tuber grades are concomitant effects of the reduced blight intensity and reduced $\mathrm{A}$ values. The increase in the tuber yield and improvement in tuber grades as a result of reduced blight intensity has been reported by several workers (Thind et al., 2004) ${ }^{[14]}$. This reduction in disease severity is achieved through the application of the effective fungitoxicants. The present results were also in consonance with their findings. From our studies, it can be concluded that seed dressing with fungicides not only helps to delay the late blight symptom development but also reduce the disease intensity. Postsymptomatic sprays of systemic fungicides applied at ten days interval after symptom development till dehaulming stage were found highly effective in the management of late blight disease intensity, spread apart from increasing the quality, size and yield of tubers. We finally conclude that integrating chemical control disease strategies along with cultural, organic and biological control methods helps to prevent the development of new races of the pathogen due to the lesser chance of resistance development against the available fungicides and also minimize the hazardous and toxic chemical residual effect on the environment.

\section{References}

1. Anonymous. Production recommendation for vegetables (Kashmir Division). Agriculture Technology Information Centre. Directorate of Extension Education. Sher-eKashmir University of Agricultural Sciences and Technology of Kashmir, 2004, 9.

2. Chakraborty A, Mazumdar D. Development of effective spray schedule for the management of late blight of potato in plains of west Bengal. Potato J. 2012; 39(1):9294.

3. Gomez KA, Gomez AA. Statistical Procedures for Agriculture Research. International Rice Research Institute. John Willy and Sons, New York, Chickester, Brisbane, Torento, Singapore, 1984, 643.

4. Kankwatsa P, Adipala E, Hakiza JJ, Olanya M, Kidanemariam HM. Effect of integrating planting time, fungicide application and host resistant on potato late blight development in South-Western Uganda. Journal of Phytopathology 2002; 150(4, 5):248-257.

5. Khadka RB, Rawal R, Marasaini M, Shrestha GK, Chapagain T, Gautam IP. Evaluation of Different Fungicidesand Clones against Potato Late Blight (Phytophthora infestans) In Western Nepal. 5th International Conference on Agriculture, Environment and Biological Sciences (ICAEBS-16), Pattaya (Thailand), 2016.

6. Mohan C, Thind TS. Resistance and relative performance of some new fungicides for active management of potato late blight in Punjab. Indian Journal of Mycology and Plant Pathology. 1999; 29(1):23-37.

7. Muthusamy M. M.Sc. (Ag.) Thesis, Tamil Nadu Agricultural University, Coimbatore, India, 1972.

8. Nene YL, Thapliyal. Fungicides in Plant Disease Control (3rd edition). Oxford and IBH Publishing Co. Pvt. Ltd, New Delhi, 2002.
9. Peerzada SH, Viswanath HS, Bhat KA. In-vitro studies on the effect of fungicides against mycelial growth and sporangial germination of Phytophthora infestans (Mont) de Bary causing late blight of potato. International Journal of Chemical Studies; 2020a; 8(1):2069-2075.

10. Peerzada SH, Viswanath HS, Bhat KA. Effect of foliar sprays of different protectant fungicides in curbing the menace of late blight (Phytophthora infestans) disease besides fostering tuber size and yield of potato crop. The Pharma Innovation Journal. 2020b; 9(3):606-613.

11. Porter LD, Cummings TF, Johnson DA. Effects of SoilApplied Late Blight Foliar Fungicides on Infection of Potato Tubers by Phytophthora infestans. Plant Disease 2006; 7:694-698.

12. Shaanner G, Finney RF. The effect of nitrogen fertilization on the expression of slow mildewing resistance in Knox wheat. Phytopathology. 1977; 67:1051-1056

13. Siddique NA, Aktar MS, Swapon NH. Comparative Efficacy of Different Fungicides against Late Blight Diseases of Potato incited by Phytophthora infestans (Mont.) de Bary and its Management. J Plant Pathol Microbiol. 2016; 7:364.

14. Thind TS, Mohan C, Arora JK, Raj P. Potential of some recently developed fungicides and their combination products for effective management of late blight of potato. Indian Phytopathology. 2004; 57(1):95-98.

15. Viswanath HS, Rather RA, Bhat KA, Peerzada SH. Management of post-harvest bacterial soft rot of potato caused by Pectobacterium carotovorum using bacterial bio-agents. The Pharma Innovation Journal. 2020; 9(4):31-35. 